\title{
P7 1 EFFECT OF DELETION OF CARTILAGE OLIGOMERIC MATRIX PROTEIN (COMP) ON COLLAGEN METABOLISM IN MURINE BRACHIOCEPHALIC ARTERY ATHEROSCLEROTIC LESIONS
}

doi:10.1136/heartjnl-2012-303148a.12

${ }^{1} \mathrm{~A}$ R Bond, ${ }^{* 2} \mathrm{~A}$ Hultgårdh, ${ }^{2} \mathrm{U}$ Rauch, ${ }^{1} \mathrm{C} \mathrm{L}$ Jackson. ${ }^{1}$ Bristol Heart Institute, School of Clinical Sciences, University of Bristol, Level 7, Bristol Royal Infirmary, Bristol BS2 8HW, UK; ${ }^{2}$ Department of Experimental Medical Science, Lund University, Lund, Sweden

Cartilage oligomeric matrix protein (COMP) is commonly found in bone and cartilage, but has also been detected in the extracellular matrix of atherosclerotic plaques. COMP is thought to act as a catalyst in collagen fibrillogenesis, and is predicted to be involved in plaque formation.

Apolipoprotein E (ApoE) knockout mice, fed a fatty diet, develop atherosclerotic lesions in the brachiocephalic artery (BCA). ApoE single knockout mice $(n=20)$ and ApoE/COMP double knockout mice $(n=23)$ were investigated to assess the effect of COMP deletion on atherosclerosis. Picrosirius red staining was used to stain collagen fibres and, by analysis of birefringence, to distinguish recently synthesised, thinner collagen fibres from older thicker fibres.

COMP deletion resulted in a significantly increased plaque:media area ratio and a decrease in lumen area. The number of buried fibrous layers, an indicator of previous plaque rupture, did not change. Total collagen, and the fractional content of older collagen fibres, were increased in both plaque and media $(p<0.05)$ in double knockout mice, but newly formed thin fibres were decreased in the media.

Brachiocephalic arteries expansively remodel in response to increased plaque burden, thus maintaining lumen integrity. The decreased lumen size shown here suggests that in mice lacking COMP this mechanism is impaired. Accumulation of older collagen fibres, and the diminution of newer fibres, suggest that COMP has roles in both fibrillolysis and fibrillogenesis, and that manipulation of the COMP pathway may have potential therapeutic benefits. 